\title{
Gamma Knife radiosurgery for vestibular schwannomas: evaluation of tumor control and its predictors in a large patient cohort in The Netherlands
}

\author{
Stijn Klijn, MD,1 Jeroen B. Verheul, MD, PhD, ${ }^{1}$ Guus N. Beute, MD, ${ }^{1}$ Sieger Leenstra, MD, PhD, ${ }^{1,2}$ \\ Jef J. S. Mulder, MD, PhD, ${ }^{3}$ Henricus P. M. Kunst, MD, PhD, ${ }^{3}$ and Patrick E. J. Hanssens, MD ${ }^{1}$ \\ ${ }^{1}$ Gamma Knife Center Tilburg, St. Elisabeth Hospital, Tilburg; ${ }^{2}$ Department of Neurosurgery, Erasmus Medical Center, \\ Rotterdam; and ${ }^{3}$ Department of ENT, Radboud University Medical Center, Nijmegen, The Netherlands
}

\begin{abstract}
OBJECTIVE The authors of this study sought to assess tumor control and complication rates in a large cohort of patients who underwent Gamma Knife radiosurgery (GKRS) for vestibular schwannoma (VS) and to identify predictors of tumor control.

METHODS The records of 420 patients treated with GKRS for VS with a median marginal dose of 11 Gy were retrospectively analyzed. Patients with neurofibromatosis Type 2 or who had undergone treatment for VS previously were excluded. The authors assessed tumor control and complication rates with chart review and used the Cox proportional hazards model to identify predictors of tumor control. Preservation of serviceable hearing, defined as Gardner-Robertson Class I-II, was evaluated in a subgroup of 71 patients with serviceable hearing at baseline and with available followup audiograms.
\end{abstract}

RESULTS The median VS tumor volume was $1.4 \mathrm{~cm}^{3}$, and the median length of follow-up was 5.1 years. Actuarial 5and 10 -year tumor control rates were $91.3 \%$ and $84.8 \%$, respectively. Only tumor volume was a statistically significant predictor of tumor control rate. The tumor control rate decreased from $94.1 \%$ for tumors smaller than $0.5 \mathrm{~cm}^{3}$ to $80.7 \%$ for tumors larger than $6 \mathrm{~cm}^{3}$. Thirteen patients (3.1\%) had new or increased permanent trigeminal nerve neuropathy, 4 $(1.0 \%)$ had new or increased permanent facial weakness, and $5(1.2 \%)$ exhibited new or increased hydrocephalus requiring a shunting procedure. Actuarial 3 -year and 5 -year hearing preservation rates were $65 \%$ and $42 \%$, respectively.

CONCLUSIONS The 5 -year actuarial tumor control rate of $91.3 \%$ in this cohort of patients with VS compared slightly unfavorably with the rates reported in other large studies, but the complication and hearing preservation rates in this study were similar to those reported previously. Various factors may contribute to the observed differences in reported outcomes. These factors include variations in treatment indication and in the definition of treatment failure, as well as a lack of standardization of terminology and of evaluation of complications. Last, differences in dosimetric variables may also be an explanatory factor.

http://thejns.org/doi/abs/10.3171/2015.4.JNS142415

KEY WORDS vestibular schwannoma; Gamma Knife radiosurgery; tumor control; complications; tumor volume; dosimetry; stereotactic radiosurgery

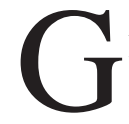
AMMA Knife radiosurgery (GKRS) has become a widely accepted primary treatment modality for small- to medium-size vestibular schwannomas (VSs). Although rates of tumor control after radiosurgery are comparable to those after microsurgery, the advantages of radiosurgery, apart from being a less invasive procedure, include better preservation of cranial nerve $(\mathrm{CN})$ function and of serviceable hearing. ${ }^{11,20}$ In the early radiosurgery era, marginal radiation doses as high as 20-70 Gy were applied. ${ }^{21}$ This dosage was gradually reduced to 12-13 Gy, after it was shown that lower marginal doses achieved equivalent tumor control but resulted in significantly lower complication rates. ${ }^{12,18}$

The first GKRS treatment for VS in the Gamma Knife

ABBREVIATIONS CN = cranial nerve; GKRS = Gamma Knife radiosurgery; VS = vestibular schwannoma .

SUBMITTED October 21, 2014. ACCEPTED April 6, 2015.

INCLUDE WHEN CITING Published online October 2, 2015; DOI: 10.3171/2015.4.JNS142415. 
Center in Tilburg, The Netherlands, was performed in July 2002. This center serves as a nationwide tertiary referral hospital. The main indication to treat patients with VS is a small- to medium-size tumor with documented growth. When serviceable hearing is present but declining, treatment is advised even in the absence of documented tumor growth. For larger tumors, however, a well-defined volumetric cut-off for the decision to treat with radiosurgery or microsurgery does not exist. Generally, VS tumors are accepted for radiosurgery if the tumor volume is below $10 \mathrm{~cm}^{3}$, unless symptoms of mass effect are present, and microsurgery is advised if the tumor is larger than 10 $\mathrm{cm}^{3}$. However, such large tumors are incidentally treated with radiosurgery when microsurgery is contraindicated because of comorbidities or high age, or when hearing preservation is of paramount importance. Until late 2010, we prescribed a dose of 11-13 Gy to the isodose covering $90 \%$ of the tumor volume, ensuring a marginal dose of approximately $11 \mathrm{~Gy}$.

The purpose of this study was to assess tumor control and complication rates in our cohort of patients treated from 2002 to 2010 at our center and to compare the results obtained with those from other large cohorts reported in the literature. In addition, we sought to identify significant predictors of VS tumor control.

\section{Methods \\ Patient Cohort}

Between July 1, 2002, and November 1, 2010, we performed 604 consecutive GKRS procedures in patients with VS. The study cohort consisted of all VS patients remaining after excluding the following: patients with neurofibromatosis Type $2(\mathrm{n}=33)$, those previously treated for VS $(n=139)$, and patients for whom follow-up data were unavailable $(\mathrm{n}=12)$. In total, 420 patients who underwent GKRS as primary treatment for unilateral VS remained eligible, and their records were retrospectively analyzed.

The treatment indications in these patients were the following: evidence of radiological progression in 237 patients (56.4\%), and in 114 patients $(27.1 \%)$, the tumor was large at initial presentation (typically, when the tumor touched the brainstem), and a wait-and-scan approach was not considered appropriate. Thirty-seven patients $(8.8 \%)$ with small but stable tumors were treated because they still had serviceable hearing, and we assumed that proactive treatment would increase their chances of maintaining functional hearing. Ten patients $(2.4 \%)$ preferred active treatment to a wait-and-scan strategy, even though treatment was not (yet) advised. In 22 patients (5.2\%), the treatment indication was not specified.

\section{Treatment and Follow-Up Protocols}

For dose planning, T1-weighted MRI scans with and without $\mathrm{Gd}$ administration $(0.1 \mathrm{mg} / \mathrm{kg}$ body weight $)$ as well as T2-weighted MRI scans were obtained. The slice thickness was usually 1 or $2 \mathrm{~mm}$ with no slice gap. In 3 patients with contraindications for MRI, CT images were taken instead. The target volume was defined as the contrast-enhancing lesion. Until November 2008, 286 patients were treated with Leksell Gamma Knife 4C (Elekta AB); thereafter, 134 patients were treated with Leksell Gamma Knife Perfexion (Elekta AB).

Depending on tumor size and whether the patient still had serviceable hearing, a dose of 11-13 Gy was prescribed to the isodose covering $90 \%$ of the tumor volume, ensuring a marginal dose of approximately $11 \mathrm{~Gy}$. In this study, the marginal dose is defined as the dose to $100 \%$ of the tumor volume. For the $4 \mathrm{C}$ model, dose calculations were performed with GammaPlan Wizard version 5.32 or Leksell GammaPlan 4C. For the Perfexion model, dose calculations were performed with Leksell GammaPlan version 8.3.1 or version 9.0.0. Follow-up imaging was performed within a standard interval of 1 year, which was often either shortened or extended according to current individual tumor size and control prognosis. Linear (2D) tumor size measurement was performed on the treatment and follow-up T1-weighted, Gd-enhanced MRI studies. In addition, for the purpose of this study, we measured volumetric (3D) tumor size. Tumors were defined as cystic if a significant spherical or oval-shaped mass was present that appeared hypointense on the T1-weighted images and hyperintense on the T2-weighted images. A neurosurgeon or radio-oncologist conducted a patient interview and neurological examination shortly before treatment and after every follow-up scan.

\section{Collection of Study Data}

All patient charts were reviewed to assess tumor control and complication rates. The tumor control rate was defined as $100 \%$ minus the fraction of cases in which treatment had failed. Treatment failure was defined as the requirement for additional treatment with either secondary GKRS, microsurgery, or a combination of the 2 procedures. We did not include shunting procedures for hydrocephalus in the definition of treatment failure.

The main indication for additional treatment was obvious tumor progression noted at consecutive measurements. We did not follow a uniform definition of progression, but we generally adhered to a minimum diameter increase of $2 \mathrm{~mm}$ in any direction as an indicator for progression. The final decision whether a patient required additional treatment was made in a multidisciplinary session involving at least a neurosurgeon, a radiation oncologist, and a radiologist. In all cases of radiological progression, a transient, radiation-induced swelling was taken into consideration and ruled out by evaluation of the tumor size on consecutive MRI scans.

Complications were defined as new or increased permanent $\mathrm{CN}$ symptoms (trigeminal numbness, paresthesia or pain/neuralgia; facial weakness reported as HouseBrackmann grade; or tinnitus or vertigo) or hydrocephalus requiring a shunting procedure.

Audiograms before and after treatment were available for 71 patients who had serviceable hearing before the treatment. The audiograms were evaluated according to the Gardner-Robertson classification. ${ }^{6}$ Serviceable hearing was defined as Gardner-Robertson Class I-II (speech discrimination $>50 \%$ and pure tone average $<50 \mathrm{~dB}$ ).

\section{Statistical Analysis}

Normal distribution of the data for each variable was vi- 
sually inspected and tested with the Kolmogorov-Smirnov test. Data with a normal distribution are presented as mean $\pm \mathrm{SD}$, whereas data that were not normally distributed are presented as median and interquartile range. The product limit method of Kaplan and Meier ${ }^{10}$ was used to calculate actuarial tumor control and hearing preservation rates. Differences among strata were tested for statistical significance with the log-rank test. To test for possible predictors of tumor control, univariate and stepwise (forward conditional) multivariate analyses were performed using the Cox proportional hazards model. The following candidate predictor variables were tested: patient age, whether the tumor was cystic or not, tumor volume, and marginal radiation dose. A p value of $<0.05$ was considered statistically significant.

\section{Literature Search}

Literature searches were performed on PubMed with the following query: ("neuroma, acoustic"[Mesh] OR vestibular schwannoma) AND ("radiosurgery"[Mesh] OR stereotactic OR Gamma Knife) AND (tumor control OR outcome OR results OR follow-up OR long-term OR complications OR dose). Search filters were set to English, "humans," and publication date until March 1, 2015. The relevance of the articles retrieved was assessed by reading the titles, abstracts, or both. Articles were excluded if the study did not represent original research, the number of patients receiving GKRS as primary treatment for sporadic VS was $<100$, dosimetry data (including marginal dose) was not available, data on tumor control at least 5 years posttreatment (actuarial or crude) was not available, or if we were unable to access the full text of the study. To minimize overlap in the patient cohorts presented, if several publications from the same authors or center were eligible for inclusion, we selected that study whose methods and design were most similar to ours (such as, for example, in the number of patients, follow-up length, etc.).

\section{Results}

\section{Descriptive Statistics}

The descriptive statistics of the characteristics of our patient cohort are presented in Table 1, and a summary of the GKRS dosimetry data used in this study is shown in Table 2. Data on symptoms arising from CN VIII (except hearing) before and after the treatment were available for 371 patients $(88.3 \%)$.

\section{Tumor Control}

The GKRS treatment controlled the tumor in 375 patients (89.3\%), and 45 patients (10.7\%) required additional treatment (Fig. 1). Among the patients in the latter group, $32(7.6 \%)$ received additional GKRS, and 13 (3.1\%) required microsurgery. The actuarial 5- and 10-year tumor control rates were $91.3 \%$ and $84.8 \%$, respectively.

The median length of time between the first and the second treatment was 4.2 years (range 1.6-7.0 years) for patients requiring additional GKRS, and 3.1 years (range 0.3-5.1 years) for microsurgery. The indication for additional treatment was progression of the solid part of the tumor in 42 patients (10.0\%), progression of the cystic part
TABLE 1. Descriptive statistics of patient and tumor characteristics in this study*

\begin{tabular}{|c|c|}
\hline Variable & Value \\
\hline Mean patient age in yrs (range) & $57.6 \pm 12.7(15-86)$ \\
\hline $\mathrm{M} / \mathrm{F}$ sex ratio (\%) & $218(52): 202(48)$ \\
\hline \multicolumn{2}{|l|}{ Tumor location (no. of pts [\%]) } \\
\hline Right & $224(53)$ \\
\hline Left & $196(47)$ \\
\hline Median tumor vol in $\mathrm{cm}^{3}$ (IQR [range]) & $1.4(0.59-3.7[0.01-17.7])$ \\
\hline \multicolumn{2}{|l|}{ Cystic tumor (no. of pts [\%]) } \\
\hline Yes & $30(7)$ \\
\hline No & $390(93)$ \\
\hline \multicolumn{2}{|l|}{ Cystic part irradiated (no. of pts [\%]) } \\
\hline Yes & $25(83)$ \\
\hline No & $5(17)$ \\
\hline Median length of FU in yrs (IQR [range]) & $5.1(4.0-7.0[0.2-12.0])$ \\
\hline \multicolumn{2}{|l|}{ Long-term FU (no. of pts [\%]) } \\
\hline$\geq 2 \mathrm{yrs}$ & $405(96)$ \\
\hline$\geq 4$ yrs & $330(79)$ \\
\hline$\geq 6$ yrs & $169(40)$ \\
\hline$\geq 8 \mathrm{yrs}$ & $68(16)$ \\
\hline$\geq 10$ yrs & $22(5)$ \\
\hline \multicolumn{2}{|l|}{$\begin{array}{l}\text { Preexisting CN V neuropathy (no. of pts } \\
{[\%] \text { ) }}\end{array}$} \\
\hline Trigeminal numbness \&/or paraesthesia & $59(14.0)$ \\
\hline Trigeminal pain/neuralgia & $6(1.4)$ \\
\hline \multicolumn{2}{|l|}{$\begin{array}{l}\text { Preexisting CN VII neuropathy (no. of } \\
\text { pts [\%]) }\end{array}$} \\
\hline $\begin{array}{l}\text { Subjective facial weakness (not objec- } \\
\text { tively assessed on physical exam) \&/or } \\
\text { transient/intermittent facial spasm }\end{array}$ & $10(2.4)$ \\
\hline $\begin{array}{l}\text { Objectively assessed (on physical exam) } \\
\text { facial weakness (HB Grade } \geq I I)\end{array}$ & $12(2.9)$ \\
\hline \multicolumn{2}{|l|}{$\begin{array}{l}\text { Preexisting CN VIII neuropathy (except } \\
\text { hearing) (no. of pts [\%]) }\end{array}$} \\
\hline Tinnitus & $277(75)$ \\
\hline Vertigo & $216(58)$ \\
\hline
\end{tabular}

$\mathrm{FU}=$ follow-up; $\mathrm{HB}=$ House-Brackmann; $\mathrm{IQR}$ = interquartile range; $\mathrm{pts}=$ patients.

* Data represent the mean \pm SD or median and IQR, depending on the normality of the data distribution.

of the tumor in 2 patients (0.5\%), and visual impairment and papilledema without radiological tumor progression in 1 patient $(0.2 \%)$. In the 44 patients with tumor progression, 2 volumetric patterns were observed. Continuous growth was observed in 27 tumors; in these tumors, the mean volume increased from 2.91 to $6.67 \mathrm{~cm}^{3}$. Initial shrinkage or stabilization of the tumor followed by regrowth was observed in 17 tumors. In these tumors, the initial mean volume was $4.38 \mathrm{~cm}^{3}$, and the mean volume before retreatment was $4.03 \mathrm{~cm}^{3}$.

The potential predictors of VS tumor control are shown in Table 3. Only tumor volume statistically significantly predicted rates of tumor control $(\mathrm{p}<0.01)$, and the tumor 
TABLE 2. Summary overview of the dosimetry parameters in this study

\begin{tabular}{ll}
\hline \multicolumn{1}{c}{ Dose } & \multicolumn{1}{c}{ Median (IQR [range]) } \\
\hline Prescription isodose in \% & $62(58-67[41-93])$ \\
\hline Dose to $100 \%$ of the tumor vol in Gy & $11.1(10.9-11.3[8.8-13.0])$ \\
\hline Dose to $99 \%$ of the tumor vol in Gy & $11.5(11.3-11.8[10.0-14.8])$ \\
\hline Dose to $95 \%$ of the tumor vol in Gy & $12.4(12.1-12.6[11.3-15.6])$ \\
\hline
\end{tabular}

control rates stratified by the volume of the tumor are shown in Fig. 2. The actuarial 5-year tumor control rate was statistically significantly higher in tumors $<0.5 \mathrm{~cm}^{3}$ (94.1\%) than in tumors $\geq 0.5 \mathrm{~cm}^{3}(90.0 \%)$ (log-rank test, $\mathrm{p}<0.05$ ). In addition, the actuarial 5-year tumor control rate was statistically significantly higher in tumors $<6$ $\mathrm{cm}^{3}(92.2 \%)$ than in tumors $\geq 6 \mathrm{~cm}^{3}(80.7 \%)$ (log-rank test, $\mathrm{p}<0.05)$.

\section{Complications}

Thirteen of the patients (3.1\%) had new or increased permanent trigeminal nerve neuropathy. Four patients $(1.0 \%)$ had new or increased permanent facial weakness reported as a decrease in House-Brackmann grade (1 patient with Grade II, 2 with Grade IV, and 1 with Grade V). Five patients (1.2\%) had new or increased hydrocephalus requiring a shunting procedure. In the 371 patients with available data on symptoms arising from CN VIII, new or increased symptoms of tinnitus were reported by 49 patients $(13.2 \%)$, and new or increased symptoms of vertigo were noted by 55 patients $(14.8 \%)$. The results for the rate of hearing preservation after GKRS among the 71 evaluable patients during the study period are shown in Fig. 3; in 43 of these patients $(60.6 \%)$, serviceable hearing was preserved. The actuarial 3- and 5-year hearing preservation rates were $65 \%$ and $42 \%$, respectively.

\section{Literature Search}

The query string described in Methods yielded 733 articles indexed in PubMed. After applying our exclusion criteria, 8 articles remained in our literature-based analysis. ${ }^{1,3,5,7,13,15,19,23}$ The tumor control and complication rates in these published cohorts are summarized in Table 4.

\section{Discussion}

The actuarial 5-year tumor control rate with GKRS in our study was $91.3 \%$, which was lower than rates of $93 \%$ $97 \%$ tumor control reported by other large studies. $5,13,19,23$ However, $97 \%$ of our patients did not require microsurgery, which is well within the range of the 95\%-99\% rates observed in other studies., ${ }^{3,13,15,19}$ The $\mathrm{CN}$ complication and hearing preservation rates we observed here do not appear to be different from those reported in previous studies. ${ }^{3,5,7,13}$ In the following, we will discuss several possible explanations for these findings.

\section{Selection of Patients}

Opinions concerning if and when a patient with VS should undergo GKRS have changed over time. As the

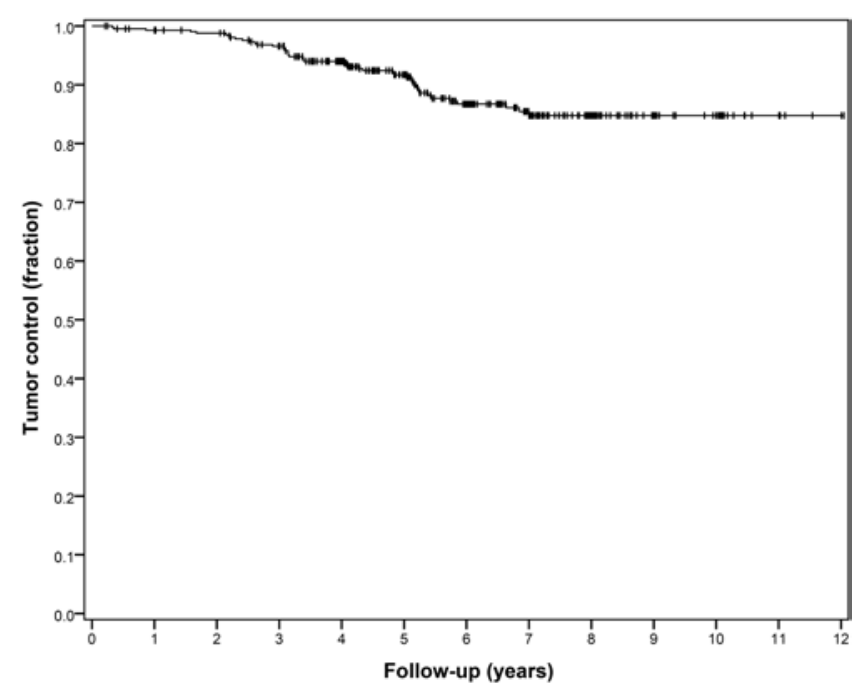

FIG. 1. Kaplan-Meier curve of actuarial tumor control rates over time in the patients of this study. Tick marks indicate censored cases.

quality of planning improved, the treatment of larger tumors became more feasible. The decisions about the highest tumor volume that is considered eligible for GKRS may still differ among different centers. In the current study, we found that tumor volume is a statistically significant predictor of tumor control with GKRS, and this relationship was not confounded by the nature (cystic versus noncystic) of the tumor. Furthermore, centers treating only tumors that show radiological evidence of progression may obtain less favorable outcomes than centers ${ }^{3,15}$ that also treat stable tumors. However, previous results in a subgroup analysis of 100 patients suggest that documented tumor growth does not have an effect on tumor control. ${ }^{25}$

\section{Comparability of Studies}

We note that many factors preclude a reliable comparison of our results with findings reported from other studies, and we discuss these factors in the following.

\section{Actuarial Tumor Control Is Often Not Calculated}

Some reports present only crude figures of tumor control, ${ }^{8,16,22}$ hampering a reliable comparison among studies.

\section{Definitions of Treatment Failure and Complications Are Not Uniform Among Studies}

In this study, treatment failure was defined as a requirement for additional treatment with secondary GKRS, microsurgery, or a combination of these procedures. Shunting procedures for hydrocephalus were not included in this definition and were instead considered a complication. Many other studies have provided different or unclear definitions of treatment failure and complications. Some studies define treatment failure as the requirement for microsurgical tumor resection and do not mention whether the need for a secondary GKRS is considered a treatment failure ${ }^{3,4}$ Other studies have defined tumor control as the absence of radiologically identified progression. ${ }^{15,16,28}$ Two studies counted cases in which patients underwent shunting procedures for hydrocephalus as treatment failures. ${ }^{14,23}$ 
TABLE 3. Candidate variables predicting tumor control

\begin{tabular}{|c|c|c|c|c|}
\hline \multirow[b]{3}{*}{ Predictor } & \multicolumn{4}{|c|}{ Analysis Type } \\
\hline & \multicolumn{2}{|c|}{ Univariate } & \multicolumn{2}{|c|}{ Multivariate } \\
\hline & Hazard Ratio & p Value & Hazard Ratio* & $p$ Value \\
\hline Patient age & 0.99 & 0.31 & - & 0.48 \\
\hline Cystic/noncystic tumor & 1.43 & 0.50 & - & 0.88 \\
\hline Tumor vol† & 1.11 & $<0.01$ & 1.11 & $<0.01$ \\
\hline Marginal dose & 0.83 & 0.56 & - & 0.49 \\
\hline
\end{tabular}

To illustrate the importance of these differences in definition, we recalculated what our tumor control results would have been had we defined treatment failure as a $10 \%$ increase in tumor volume. In this scenario, the crude tumor control rate dropped from $89.3 \%$ to $77.8 \%$. We also note that volumetric follow-up is much more sensitive for detecting tumor progression than 2D (linear) follow-up. ${ }^{27}$

We encountered similar problems with varying definitions among studies while attempting to compare our complication rate with those reported from other studies. For example, a diagnosis of CN complications depends on the subjective assessment of symptoms by both the patient and the clinician. Although the diagnosis of facial weakness is usually straightforward, objectively rating complications such as pain, vertigo, and tinnitus is more challenging. Moreover, there often is no consensus about which grading scale should be used, provided that one exists for a given symptom. Thus, uniformity in reporting these complications is lacking. Furthermore, it appears that hearing preservation after radiosurgery continues to decline. ${ }^{2}$ Studies on hearing preservation rates should therefore report actuarial instead of crude figures for serviceable hearing, especially in the long term.

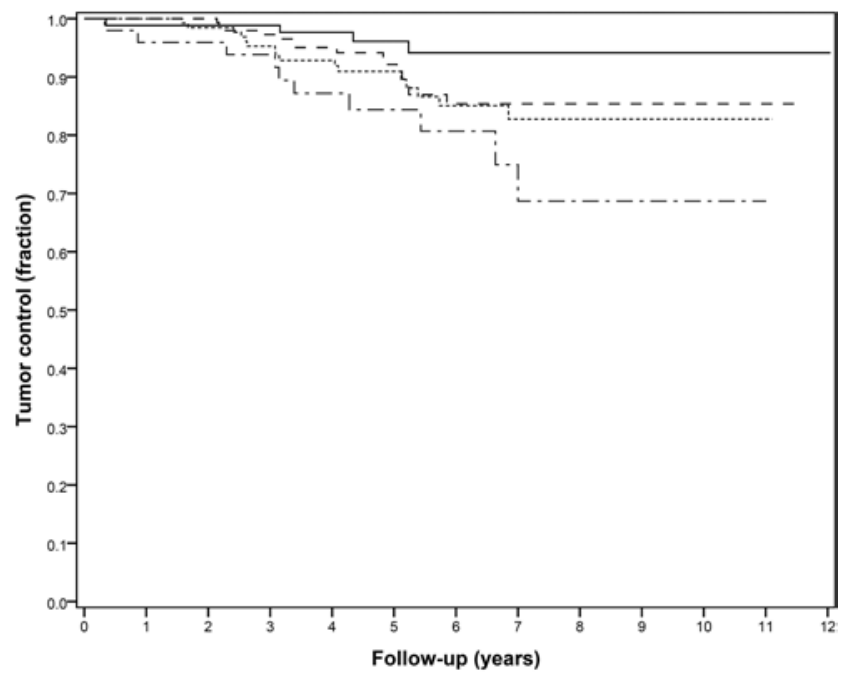

FIG. 2. Kaplan-Meier curves of actuarial tumor control rates, stratified by tumor volume. The continuous line indicates a tumor volume of $<0.50$ $\mathrm{cm}^{3}$; long dashes depict a tumor volume of $0.50-1.99 \mathrm{~cm}^{3}$; short dashes correspond to a tumor volume of $2.00-5.99 \mathrm{~cm}^{3}$; and alternating long and short dashes indicate a tumor volume of $\geq 6 \mathrm{~cm}^{3}$.
The Requirement for Additional Treatment Is Not Based on Uniform Criteria

Although tumor progression is the indication for additional treatment in nearly all cases of VS, the decision if and when a clinician should proceed with additional treatment is inevitably a matter of judgment by different clinicians in different centers. Such decisions may depend on many patient-related and tumor-related variables such as patient age and comorbidities, the method of assessing tumor size (i.e., linear vs volumetric measurement), the magnitude of radiological progression that is considered significant and clinically relevant, and the time frame during which transient radiation-induced swelling is taken into consideration. Obviously, the relative importance of these and other variables is strongly influenced by tumor size. Although we attempted to describe our workup as clearly as possible, it is unlikely that clinicians from other centers would have made exactly the same decisions for all patients in our cohort.

The aforementioned issues illustrate the importance of guidelines and standardization of terminology in stereotactic radiosurgery as proposed by the Standardization Committee of the International Leksell Gamma Knife Society. ${ }^{26}$ Such standardization will also enable the com-

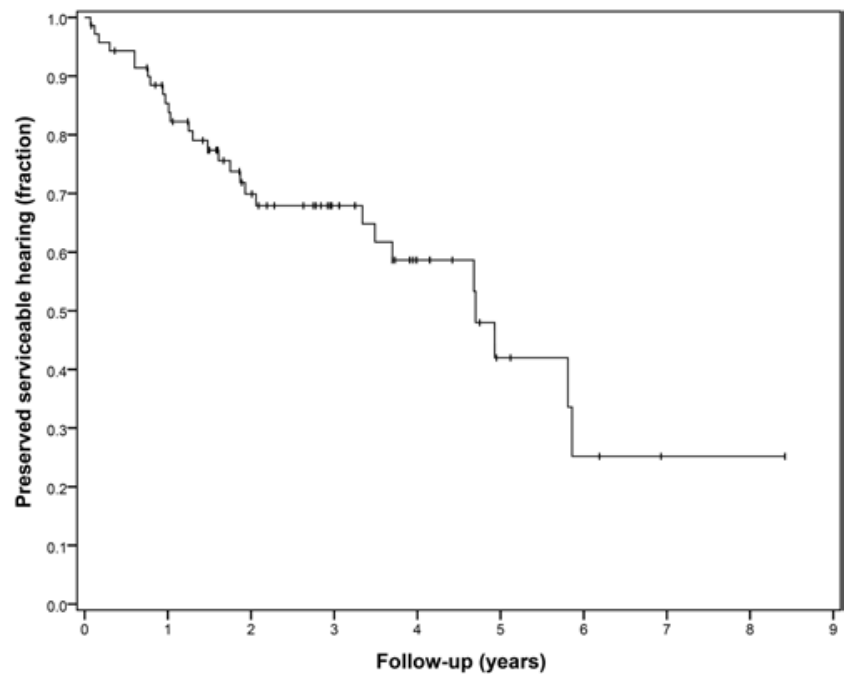

FIG. 3. Kaplan-Meier curve of actuarial preservation of serviceable hearing (i.e., Gardner-Robertson Class I-II) among the patients of this study. Tick marks indicate censored cases. 


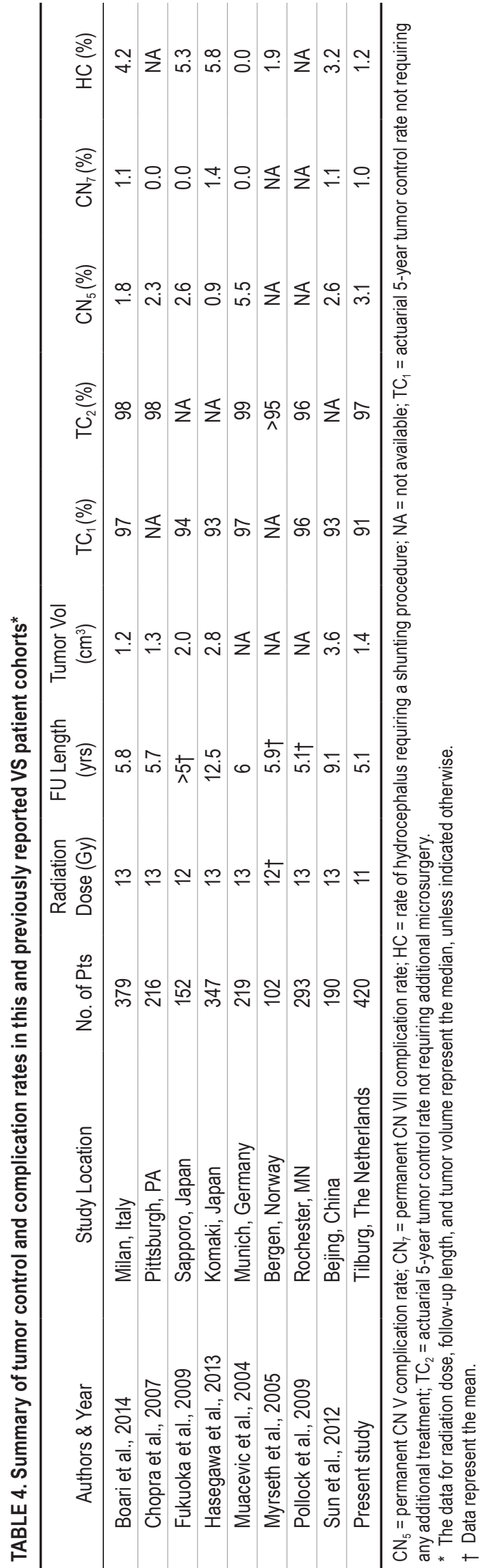

parison of outcomes after stereotactic radiosurgery with different treatment modalities. Another aspect that should be standardized is visualization and delineation of the target and relevant critical structures such as the cochlea. This project is currently underway within the International Leksell Gamma Knife Society.

\section{Marginal Dose}

Assuming that patient and tumor selection, definitions of outcome variables, and length of follow-up are perfectly comparable among the previous cohort studies and that our tumor control rate was therefore truly lower, a marginal dose of 11 Gy may be too low to achieve an optimal tradeoff between tumor control and complication rates. In the early years of GKRS, after many authors had observed that high marginal doses produce significant $\mathrm{CN}$ complications, the marginal dose was gradually decreased to reduce such complications. At present, most centers use marginal doses between 12 and 13 Gy without an apparent decline in tumor control. ${ }^{12,18}$ Since the first treatment at our center in 2002, we have prescribed a dose of 11-13 Gy to the isodose covering $90 \%$ of the tumor volume, ensuring a marginal dose of approximately $11 \mathrm{~Gy}$. This strategy in dose prescription was adopted from experts in the field of GKRS.

In the entire cohort presented here, only 7 patients $(1.7 \%)$ received a marginal dose of 12 Gy or higher. Considering our tumor control rate, when using marginal doses below 12 Gy, a turning point may have been reached when tumor control starts to decline while complication rates do not decrease any further. In 2011, we started to prescribe a dose of 11-13 Gy to the isodose covering 99\% (instead of $90 \%$ ) of the tumor volume. We now continuously and prospectively collect data from all our patients, which will allow us in the long term to evaluate whether this change in our treatment protocol improves control of the VS tumor. If better tumor control is indeed achieved with this altered dose regimen, this would be a strong indication that a marginal dose of $11 \mathrm{~Gy}$ is too low, provided that we can keep constant all of the other variables discussed above that may influence tumor control.

\section{Study Limitations}

The patients in this study were treated over a period of $>8$ years, and indications for treatment may have changed during this time interval. During these 8 years, radiological evidence of progression has become more important in the decision to treat a VS. Furthermore, improved quality of planning and good treatment results have encouraged us now to accept tumors for GKRS that are larger than those considered for this treatment a decade ago. These changes may have influenced the outcomes in this study.

The retrospective nature of this study is a limitation. Although we are confident that important complications like facial weakness were thoroughly assessed and reported at each follow-up, it may be disputed whether measured symptoms such as, for example, tinnitus and vertigo, which are more difficult to objectively determine, can be accurately assessed on the basis of a chart review. In response to these concerns and for the purpose of future analysis, we currently use validated tinnitus and vertigo scores., ${ }^{9,17}$ 
Hearing preservation is considered one of the most important issues in present VS management. The number of patients who had audiometric follow-up was limited in this study. This limitation is due to the nature of our institution as a tertiary referral center; audiograms are performed by the referring medical centers, not by our department. For the purpose of this study, we contacted referring centers to collect these audiometric data. The criteria for performing audiometric examinations may differ among these centers, but most conduct follow-up audiometry only if the patient reports decreased hearing. This may have biased the results of this study, leading to an underestimation of hearing preservation rates. Timmer et al. ${ }^{24}$ showed an important effect of the maximal cochlear dose on hearing preservation, which we had not yet recognized at the time of treatment of the patients in this study. We now aim to keep the cochlear dose below $4 \mathrm{~Gy}$, and we expect that this lower cochlear dose will improve hearing preservation.

\section{Conclusions}

The actuarial 5-year tumor control of $91.3 \%$ in our cohort compared slightly unfavorably with those reported in other studies, but the complication and hearing preservation rates we observed here were comparable to previously observed rates. Various factors may contribute to the observed differences in some of the outcomes reported here. These factors include variations among studies in treatment indication and in the definition of treatment failure, as well as a lack of standardization of terminology and evaluation of complications. Last, differences in the dosimetric parameters may also explain some of the outcome differences. Standardization of terminology and in the assessment of complications may greatly increase the validity of future comparisons among large patient cohorts and may enable basing conclusions about outcome predictors on solid statistical procedures.

\section{References}

1. Boari N, Bailo M, Gagliardi F, Franzin A, Gemma M, del Vecchio A, et al: Gamma Knife radiosurgery for vestibular schwannoma: clinical results at long-term follow-up in a series of 379 patients. J Neurosurg 121 Suppl:123-142, 2014

2. Carlson ML, Jacob JT, Pollock BE, Neff BA, Tombers NM, Driscoll CL, et al: Long-term hearing outcomes following stereotactic radiosurgery for vestibular schwannoma: patterns of hearing loss and variables influencing audiometric decline. J Neurosurg 118:579-587, 2013

3. Chopra R, Kondziolka D, Niranjan A, Lunsford LD, Flickinger JC: Long-term follow-up of acoustic schwannoma radiosurgery with marginal tumor doses of 12 to $13 \mathrm{~Gy}$. Int J Radiat Oncol Biol Phys 68:845-851, 2007

4. Flickinger JC, Kondziolka D, Niranjan A, Maitz A, Voynov G, Lunsford LD: Acoustic neuroma radiosurgery with marginal tumor doses of 12 to 13 Gy. Int J Radiat Oncol Biol Phys 60:225-230, 2004

5. Fukuoka S, Takanashi M, Hojyo A, Konishi M, Tanaka C, Nakamura H: Gamma knife radiosurgery for vestibular schwannomas. Prog Neurol Surg 22:45-62, 2009

6. Gardner G, Robertson JH: Hearing preservation in unilateral acoustic neuroma surgery. Ann Otol Rhinol Laryngol 97:55-66, 1988

7. Hasegawa T, Kida Y, Kato T, Iizuka H, Kuramitsu S, Yamamoto T: Long-term safety and efficacy of stereotactic radio- surgery for vestibular schwannomas: evaluation of 440 patients more than 10 years after treatment with Gamma Knife surgery. J Neurosurg 118:557-565, 2013

8. Hempel JM, Hempel E, Wowra B, Schichor Ch, Muacevic A, Riederer A: Functional outcome after gamma knife treatment in vestibular schwannoma. Eur Arch Otorhinolaryngol 263:714-718, 2006

9. Jacobson GP, Newman CW: The development of the Dizziness Handicap Inventory. Arch Otolaryngol Head Neck Surg 116:424-427, 1990

10. Kaplan EL, Meier P: Non-parametric estimation and incomplete observations. J Am Stat Assoc 53:457-481, 1958

11. Kondziolka D, Lunsford LD, McLaughlin MR, Flickinger JC: Long-term outcomes after radiosurgery for acoustic neuromas. N Engl J Med 339:1426-1433, 1998

12. Lunsford LD, Niranjan A, Flickinger JC, Maitz A, Kondziolka D: Radiosurgery of vestibular schwannomas: summary of experience in 829 cases. J Neurosurg 102 Suppl:195-199, 2005

13. Muacevic A, Jess-Hempen A, Tonn JC, Wowra B: Results of outpatient gamma knife radiosurgery for primary therapy of acoustic neuromas. Acta Neurochir Suppl 91:75-78, 2004

14. Murphy ES, Barnett GH, Vogelbaum MA, Neyman G, Stevens $\mathrm{GH}$, Cohen $\mathrm{BH}$, et al: Long-term outcomes of Gamma Knife radiosurgery in patients with vestibular schwannomas. J Neurosurg 114:432-440, 2011

15. Myrseth E, Møller P, Pedersen PH, Vassbotn FS, WentzelLarsen T, Lund-Johansen M: Vestibular schwannomas: clinical results and quality of life after microsurgery or gamma knife radiosurgery. Neurosurgery 56:927-935, 2005

16. Nakaya K, Niranjan A, Kondziolka D, Kano H, Khan AA, Nettel B, et al: Gamma knife radiosurgery for benign tumors with symptoms from brainstem compression. Int J Radiat Oncol Biol Phys 77:988-995, 2010

17. Newman CW, Jacobson GP, Spitzer JB: Development of the Tinnitus Handicap Inventory. Arch Otolaryngol Head Neck Surg 122:143-148, 1996

18. Petit JH, Hudes RS, Chen TT, Eisenberg HM, Simard JM, Chin LS: Reduced-dose radiosurgery for vestibular schwannomas. Neurosurgery 49:1299-1307, 2001

19. Pollock BE, Link MJ, Foote RL: Failure rate of contemporary low-dose radiosurgical technique for vestibular schwannoma. J Neurosurg 111:840-844, 2009

20. Pollock BE, Lunsford LD, Kondziolka D, Flickinger JC, Bissonette DJ, Kelsey SF, et al: Outcome analysis of acoustic neuroma management: a comparison of microsurgery and stereotactic radiosurgery. Neurosurgery 36:215-229, 1995

21. Régis J, Roche PH, Delsanti C, Thomassin JM, Ouaknine M, Gabert K, et al: Modern management of vestibular schwannomas. Prog Neurol Surg 20:129-141, 2007

22. Roos DE, Potter AE, Brophy BP: Stereotactic radiosurgery for acoustic neuromas: what happens long term? Int J Radiat Oncol Biol Phys 82:1352-1355, 2012

23. Sun S, Liu A: Long-term follow-up studies of Gamma Knife surgery with a low margin dose for vestibular schwannoma. J Neurosurg 117 Suppl:57-62, 2012

24. Timmer FC, Hanssens PE, van Haren AE, Mulder JJ, Cremers CW, Beynon AJ, et al: Gamma knife radiosurgery for vestibular schwannomas: results of hearing preservation in relation to the cochlear radiation dose. Laryngoscope 119:1076-1081, 2009

25. Timmer FC, Mulder JJ, Hanssens PE, van Overbeeke JJ, Donders RT, Cremers CW, et al: Gamma knife radiosurgery for vestibular schwannomas: identification of predictors for continued tumor growth and the influence of documented tumor growth preceding radiation treatment. Laryngoscope 121:1834-1838, 2011

26. Torrens M, Chung C, Chung HT, Hanssens P, Jaffray D, Kemeny A, et al: Standardization of terminology in stereotactic 
radiosurgery: Report from the Standardization Committee of the International Leksell Gamma Knife Society: special topic. J Neurosurg 121 Suppl:2-15, 2014

27. van de Langenberg R, de Bondt BJ, Nelemans PJ, Baumert BG, Stokroos RJ: Follow-up assessment of vestibular schwannomas: volume quantification versus two-dimensional measurements. Neuroradiology 51:517-524, 2009

28. Wangerid T, Bartek J Jr, Svensson M, Förander P: Long-term quality of life and tumour control following gamma knife radiosurgery for vestibular schwannoma. Acta Neurochir (Wien) 156:389-396, 2014

\section{Disclosure}

The authors report no conflict of interest concerning the materials or methods used in this study or the findings specified in this paper.

\section{Author Contributions}

Conception and design: Hanssens, Klijn, Verheul. Acquisition of data: Hanssens, Klijn, Verheul. Analysis and interpretation of data: Hanssens, Klijn, Verheul, Leenstra. Drafting the article: Klijn. Critically revising the article: all authors. Reviewed submitted version of manuscript: Hanssens, Klijn. Approved the final version of the manuscript on behalf of all authors: Hanssens. Statistical analysis: Klijn. Study supervision: Hanssens.

\section{Supplemental Information Previous Presentation}

Portions of this work were presented as an oral presentation at the 10th Biennial Congress and Exhibition of the International Stereotactic Radiosurgery Society in Paris, France, May 8-12, 2011.

\section{Correspondence}

Patrick E. J. Hanssens, Gamma Knife Center Tilburg, St. Elisabeth Hospital, P.O. Box 90151, Tilburg 5000 LC, The Netherlands. email: p.hanssens@elisabeth.nl. 